\title{
LOS RECURSOS MADEREROS. CONDICIONES SOCIOECONÓMICAS VINCULADAS CON SU APROVECHAMIENTO EN LA PROVINCIA DEL CHACO
}

\section{THE TIMBER RESOURCES. SOCIO-ECONOMIC CONDITIONS ASSOCIATED WITH ITS USE IN THE CHACO PROVINCE}

\author{
Lic. Dante E. Cuadra \\ Profesor Adjunto, Cátedra Introducción a la Geografía \\ Profesor Adjunto, Cátedra Seminario de Geografía Humana \\ Investigador categoría 3 -CRC NEA \\ Dpto. de Geografía, Facultad de Humanidades, UNNE. \\ E-mail: dantecuadra@yahoo.com
}

\section{RESUMEN}

La actividad foresto-industrial atraviesa diferentes estratos sociales. Para entender su dinámica, primeramente debe conocerse su organización interna, es decir, quiénes forman parte de la misma. Cumplido este paso inicial de la investigación, se elaboró una caracterización de los componentes del sistema, enfatizándose en las condiciones socioeconómicas de cada grupo y, vinculado a ello, la situación de vulnerabilidad en la que se encuentran.

Dentro de este escenario, los ámbitos que se han reconocido como demandantes de maderas (materias primas o insumos para el desarrollo de sus actividades) y otros asociados, donde se hallan los diferentes actores que nos interesa analizar, son los siguientes: la fábrica taninera, el aserradero y la carpintería de mediana dimensión, la pequeña fábrica, el sector asalariado, el taller de pulido, el acopio y reventa, el artesanado, la producción de leña, la fabricación de postes, la producción de carbón vegetal, la empresa de servicios diversificados, la fabricación de ladrillos, el sector transportista, el proveedor de recursos forestales y los sectores potenciales (fábrica de arrabio, pellets, espirales y aglomerados). Cada uno de estos componentes admiten subcategorías que exponen diferentes rasgos y grados de vulnerabilidad social y económica, que se analizan específicamente en esta investigación, a partir de un pormenorizado trabajo de campo que permitió la observación in situ y una diversidad de entrevistas abiertas y estructuradas a los distintos actores involucrados.

\section{PALABRAS CLAVES}

Explotación forestal; industria maderera; condiciones socioeconómicas; pobreza; vulnerabilidad económica y social.

\section{SUMMARY}

Forest industrial activity passes through different social strata. To understand its dynamics, first of all you need to know its internal organization, i.e., who are part of the same. Complied with this initial step of the research, it has been developed a characterization of the components of the system, focusing on the socio-economic conditions of each group and, linked to it, the situation of vulnerability in which they are found.

In this scenario, the areas which require wood (raw materials and inputs for the development of their activities) and other related sectors, where there are different actors that we are interested in analyzing; are as follows: the tannin industry, sawmill and carpentry medium dimension, the small factory, the wage sector, polishing workshop, the storage and resale, crafts, firewood production, manufacture of poles, charcoal production, diversified services companies, the manufacture of bricks, carrier sector, the supplier of forest resources and potential sectors (pig iron, spirals, pellets, plywood factory). Each of these components support subcategories that expose different features and levels of social and economic vulnerability, which are specifically discussed in this research, based on a detailed field work which allowed the in situ observation, and a variety of interviews, some open and some structured to the various actors involved.

\section{KEY WORDS}

Forest exploitation; timber industry; socio-economic conditions; poverty; economic and social vulnerability. 
Revista Geográfica Digital. IGUNNE. Facultad de Humanidades. UNNE. Año 8. № 15. Enero - Junio 2011.

\section{Introducción}

La explotación de los bosques, particularmente los nativos, admite un amplio rango de prestaciones, que va desde la empresa multinacional hasta la recolección individual o familiar de restos maderables no utilizados a nivel comercial (leña para uso doméstico) o de desperdicios producidos por el sector industrial, que se derivan a diferentes propósitos, como pueden ser la artesanía, la producción de carbón de leña o como insumo de las fábricas de ladrillos, entre otros.

Los bosques de las zonas templadas han sido los primeros en ser afectados por la acción antrópica (particularmente Europa y, mucho más tarde, Estados Unidos o la región del espinal en Argentina, por mencionar algunos ejemplos) ante la demanda de tierras cultivables y de pastoreo, la necesidad de generar energía térmica y el requerimiento del sector artesanal e industrial para procesar sus maderas con distintos fines: construcción de viviendas, aberturas, muebles e infraestructura rural, vial y caminera o la obtención de sustancias o esencias como el tanino. Luego le tocaría el turno a las áreas tropicales, subtropicales y templado-frías, que sufrieron, desde fines del siglo XIX hasta lo que va del XXI, una fuerte presión sobre sus recursos maderables.

De acuerdo con los datos proporcionados por FAO, entre 1980 y 2010 se han talado 2 millones de $\mathrm{km}^{2}$ de bosques en América Latina, estimándose que la tasa actual de deforestación anual es de $50.000 \mathrm{~km}^{2}$.

Nuestra unidad de análisis, la provincia del Chaco, en el nordeste de Argentina, ha sufrido profundamente la explotación de sus maderas duras y semiduras desde fines del siglo XIX, sobre todo con la instalación de la empresa La Forestal, cuyo objetivo era la industrialización de rollizos de quebracho colorado (Schinopsis balansae) para la producción del tanino; pero además, la salida de otras maderas para durmientes de ferrocarril, postes, adoquines, vigas, muebles y otros usos ha sido permanente, al compás del avance de la frontera agropecuaria que necesitaba deshacerse de los bosques por la vía de la tala rasa, la venta de maderas o los incendios provocados.

Las maderas de la región chaqueña han sufrido una permanente sangría, dado que otras áreas como la pampeana, patagónica y la diagonal árida (desprovistas de árboles) han sido demandantes para la traza de sus líneas férreas, puentes, alcantarillas y miles de kilómetros de alambrados que requerían postes y varillas. Los rollos eran cortados y transportados inmediatamente, por lo que no generaron valor agregado en el lugar de origen.

Otras industrias madereras, aparte del sector taninero y de la demanda de durmientes, postes y leña, no tuvieron gran desarrollo en el Chaco; en pueblos y ciudades se levantaron aserraderos y carpinterías que, básicamente, satisfacían las demandas locales. Las actividades que lograron supremacía fueron la agricultura y en menor grado la ganadería. Pero, con la crisis agropecuaria de los años '60, sobre todo del algodón, en el centro del Chaco se ha dado un fenómeno muy particular, como ha sido la concentración de establecimientos fabriles (inicialmente pequeños talleres) que con el tiempo fueron conformando importantes parques foresto-industriales. En el resto de la provincia, la distribución de aserraderos y carpinterías fue más puntualizado y pocas veces con escalas de producción que trascendieran las demandas locales o zonales.

Al ser una actividad que traspasa diferentes niveles sociales, se puede observar que no todo los actores que allí convergen obtienen los mismos beneficios, lo que se traduce en fuertes asimetrías entre unos y otros que, en consecuencia, muestran grados de vulnerabilidad social y económica muy diferenciados.

\section{Objetivos}

- Clasificar y caracterizar los distintos sectores que componen la actividad foresto-industrial en la provincia del Chaco.

- Analizar los caracteres socioeconómicos y los grados de vulnerabilidad de los diferentes actores involucrados en dicha actividad.

\section{Información y metodología utilizadas}

-Lectura de bibliografía específica.

-Observación en el terreno (trabajo de campo).

-Consulta a informantes calificados. 
Revista Geográfica Digital. IGUNNE. Facultad de Humanidades. UNNE. Año 8. No 15. Enero - Junio 2011.

ISSN 1668-5180 Resistencia, Chaco

-Entrevistas abiertas y estructuradas a diferentes actores sociales vinculados con el tema forestoindustrial.

\section{Resultados}

La industria de la madera en la provincia del Chaco se caracteriza por ser generadora de muchos puestos de trabajo formal e informal y por no exigir una gran calificación en la mano de obra demandante. Ésta, puede clasificarse en: operarios de aserraderos y carpinterías; trabajadores independientes que realizan tareas de cepillado, lijado, pulido, ensamblado y laqueado en sus casas o talleres como un servicio a las carpinterías; artesanos en madera; fabricantes de carbón, que aprovechan la materia de descarte y, además, los ladrilleros que aprovechan el aserrín para su mezclado con la tierra, la cascarilla como base de la cancha y la leña como combustible para el horneado. Pero también debe agregarse una serie de actividades conexas que obtienen beneficios directos de estos establecimientos fabriles, como son los carreros que cargan los muebles desde las fábricas a los lugares de pulido y viceversa y trasladan los subproductos (aserrín, cascarilla, virutas, costaneros o leña) a las carbonerías y ladrillerías, los transportistas que movilizan, tanto la materia prima como los productos elaborados, los talleres mecánicos y de afilado, las ferreterías, los productores agropecuarios que venden las maderas de sus montes y las casas de comercios y servicios en general, que interceptan parte de los flujos de circulantes que generan estas actividades.

Para entender la dinámica de esta actividad económica, que atraviesa diferentes estratos sociales, debe conocerse primeramente su organización interna o estructuración, es decir cómo se integra y quiénes forman parte del mismo. Éste ha sido el primer paso de la investigación. Luego se elaboró una caracterización de los componentes del sistema foresto-industrial, enfatizándose en las condiciones socioeconómicas de cada grupo y, vinculado a ello, la situación de vulnerabilidad de cada uno.

El primer problema con el que se tuvo que lidiar fue que conocer exactamente el número de establecimientos foresto-industriales del Chaco y, particularmente del área centro chaqueña (y dentro de ella, específicamente de Machagai, localidad que alberga a la mayor cantidad de fábricas a nivel provincial), es una tarea técnicamente imposible, por las razones que se detallan a continuación:

a) Subregistro: sólo se hallan inscriptos en los organismos de jurisdicción municipales, provinciales y nacionales aquéllos aserraderos y carpinterías de mayor tamaño y volumen de procesamiento que tributan sistemáticamente (Industria y Comercio, DGR, AFIP). La absoluta mayoría de los pequeños emprendimientos existen de hecho y, por lo tanto, quienes se desempeñan en ellos lo hacen por cuenta propia (muchas veces constituyendo sociedades informales) o son empleados no registrados.

b) Elevado dinamismo de los emprendimientos de pequeño tamaño: las características que se detallan a continuación son observables en los lugares donde hay una gran concentración de la actividad industrial maderera, como ocurre en las localidades de Quitilipi, Presidencia de La Plaza y, sobre todo, Machagai, donde se emplaza el mayor número de establecimientos, recipiente de una cultura vinculada al procesamiento maderero que, en muchos casos, va por la tercera generación en materia de transmisión de saberes:

* Funcionamiento temporario de muchas de estas pequeñas fábricas, es decir que a lo largo del año se producen cierres y aperturas según las condiciones del mercado, las relaciones laborales entre los asociados, la disponibilidad de maderas en las distintas épocas, la demanda de algún comprador o la intensidad de controles que llevan a cabo los órganos administrativos e impositivos.

* Reemplazo de propietarios: un pequeño aserradero puede consistir en un techo sostenido por columnas de hierro de construcción y piso de tierra, donde se instalan dos o más máquinas de corte o aserrado, generalmente usadas, más algunos implementos y herramientas elementales. Esta característica, casi de campamento transitorio, suele ser muy frecuente, de modo que ante algún problema económico, la disolución de una sociedad de hecho u otras dificultades operativas, las instalaciones cambian de dueño o se alquilan, por lo que es notable el fenómeno de sucesión de propietarios, al tratarse de capitales con costos no muy dimensionados que pueden ser pagaderos en cuotas a lo largo de cierto tiempo convenido por las partes. 
* Fisonomía de las instalaciones: el aspecto exterior de un aserradero no refleja necesariamente cómo es su organización interna. Un galpón, desde afuera, puede dar la imagen de ser un sólo establecimiento -al no exhibir paredes divisorias en su interior-, pero en la práctica puede nuclear a dos o tres productores que realizan sus actividades junto a sus familiares o empleados informales, compartiendo el espacio, adquiriendo en conjunto la materia prima, pagando en partes iguales los costos de energía eléctrica e, incluso, haciendo un uso comunitario de máquinas y herramientas.

* Constitución de sociedades de hecho de corta duración: la conformación de estos grupos de trabajo surgen con una determinada expectativa inicial, en vistas a generar una pequeña empresa que pueda sostenerse en el tiempo. Suele estar integrada por ex empleados de aserraderos que disponen de un pequeño capital o percibieron alguna indemnización por despido o accidente, por cuentapropistas con cierta experiencia en el sector que, incluso, pueden integrar a personas que provienen de otras ocupaciones y que deciden incursionar en esta actividad. Muy a menudo, esas expectativas se esfuman y este tipo de sociedades informales duran sólo algunos meses o, en ocasiones, uno o más años: las rupturas pueden desencadenarse por diferentes factores: incompatibilidad de criterios entre los socios debido a manejos económicos sin el consenso grupal, desigual intensidad en la dedicación por parte de unos y otros o, simplemente, desavenencias derivadas de la personalidad de sus integrantes. Estas causales no son ajenas en un contexto en el que el nivel cultural suele ser entre medio y bajo, donde se hallan arraigadas ciertas costumbres en el trato interpersonal que no repercuten favorablemente en la consolidación sociológica de los grupos (chistes de mal gusto, expresiones con doble sentido, abusos de confianza o vocabulario degradante hacia sus pares), a lo que se suma el arraigo de algunos vicios como el exceso de alcohol, la falta de honestidad en el manejo de los recursos, el bajo nivel de responsabilidad ante el compromiso asumido o la actitud individualista en detrimento de sus colegas y socios).

* Alquiler de instalaciones y máquinas: ésta es una práctica muy observada y admite dos modalidades: el alquiler total, que se da cuando el dueño decide retirarse de la actividad y percibir ingresos por renta (los motivos suelen ser diversos: edad avanzada, falta de descendientes que deseen continuar al frente del establecimiento, inicio de otra actividad empresarial, saturación o cansancio tras muchos años en el rubro, enfermedad, etc.). La otra modalidad es el alquiler parcial, que consiste en un trato con la contraparte para uso de las instalaciones y máquinas en un turno del día. Por ejemplo, el propietario y su personal trabajan de 5 a 15 hs., en tanto el inquilino lo hace de 17 a 3, permitiendo un descanso para las maquinarias entre cada turno.

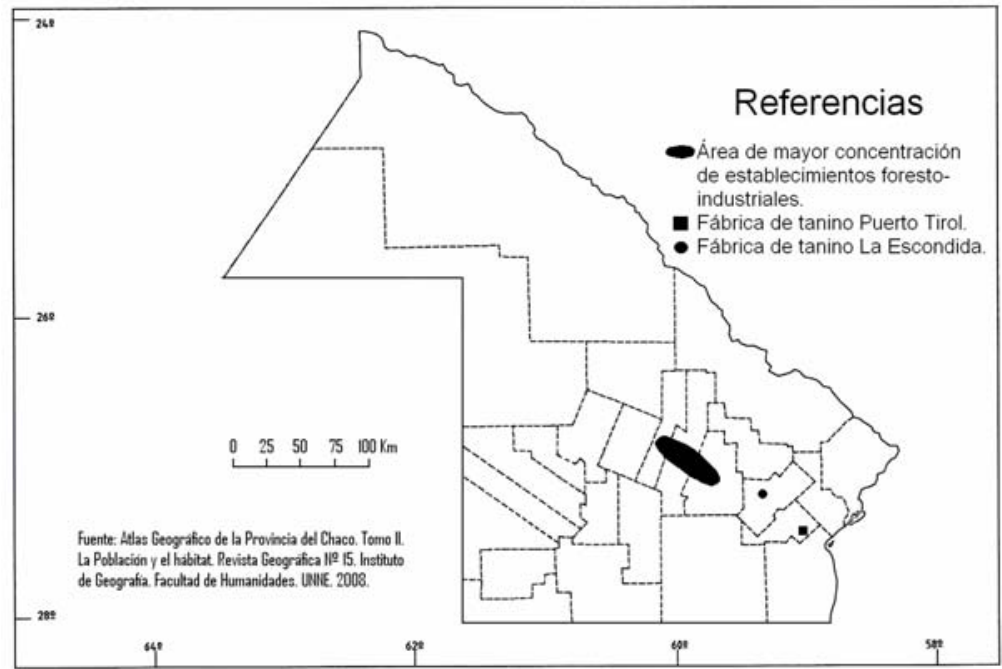

Ubicación geográfica de los establecimientos foresto-industriales más importantes del Chaco.

* Otros aspectos de la dinámica maderera, que hacen bastante dificultoso obtener datos exactos de la actividad son: el nivel de informalidad que se halla internalizado en todos los elementos del sistema, como son los préstamos de guías, la reventa de maderas de establecimientos más grandes a los más chicos, la compra grupal de rollos o rollizos (uno de ellos figura como comprador, pero la madera se 
Revista Geográfica Digital. IGUNNE. Facultad de Humanidades. UNNE. Año 8. No 15. Enero - Junio 2011.

ISSN 1668-5180 Resistencia, Chaco

destina a varios aserraderos) y la madera que se adquiere de vendedores ilegales, quienes logran sortear los mecanismos de control y descargan el producto sin guías de corte, ni autorización de transporte. Del mismo modo, es común que varios productores completen camiones o equipos con muebles, pero figura sólo uno de ellos como vendedor de toda la producción.

\section{Los tipos de establecimientos que demandan material forestal en el Chaco}

\section{La fábrica taninera}

Tradicionalmente fue la gran fábrica que se distribuía en diversos puntos de la llanura oriental del Chaco y provincias vecinas de la margen derecha del Paraná-Paraguay. Actualmente, esta industria demandante de rollizos de quebracho colorado chaqueño (Schinopsis balansae), tras un siglo de explotación, representa un estado relictual en sólo dos localidades de la provincia: Puerto Tirol (empresa UNITAN S.A.I.C.A.) y La Escondida (INDUNOR S.A.), que producen el extracto, el cual, dado el bajo consumo interno, se exporta en un 85\%. La producción obtenida se destina a diferentes usos: curtientes vegetales, dispersantes, aditivos para tableros de madera y cartones, taninos enológicos, alimentación animal, flotación de minerales, estabilizantes de suelos y taninos para la industria azucarera.

La materia prima, en este caso los rollizos de la especie mencionada, es cada vez más escasa, los costos de transportes y laborales tienden a elevarse con el paso de los años, en tanto la demanda de tanino es muy inferior a la de épocas pasadas, además de existir en el mercado otros productos alternativos de origen natural y artificial. Por estas razones, la situación de este sector es crítica en vistas a un futuro cercano: las maquinarias presentan un alto grado de obsolescencia tecnológica y tampoco es viable la inversión en su mejoramiento, dadas las perspectivas del mercado, la reducción de la riqueza forestal, la carencia histórica de planes de forestación y de reforestación que aseguraran la renovabilidad del recurso. Además, los efectos ambientales negativos generados por esta actividad, como es la fuerte y gradual reducción de áreas explotables con la especie Schinopsis balansae y la recurrente contaminación generada por las fábricas en suelos, aguas y aire a lo largo de su existencia (con las nuevas legislaciones, a partir de los años '90, se han logrado avances en materia de protección de suelos y aguas, pero sigue siendo un gran problema la emisión de humos tóxicos a la atmósfera).

El número de operarios de estas fábricas es muy acotado y se hallan agremiados en FATITA (Federación Argentina de Trabajadores Industriales del Tanino y afines) o lo hacen en sindicatos adheridos a ésta, como son SOITA (Sindicato Obrero de la Industria del Tanino) y STITA (Sindicato de Trabajadores de la Industria del Tanino y Afines). El operario típico de la empresa taninera es la de un asalariado industrial, es decir, un empleado de clase media a medio baja, con cobertura social y aportes jubilatorios. Ello no implica que la empresa no tenga personal contratado e, inclusive, no registrado para ciertas labores específicas, secundarias o transitorias. El perfil sindicalizado de los operarios constituye una autoprotección ante decisiones o medidas implementadas por la empresa que pudieran perjudicarlos (despidos, jornadas reducidas, suspensiones u otras sanciones). El personal de planta, en su gran mayoría dispone de vivienda propia, satisface las necesidades básicas de su grupo familiar y tiene beneficios adicionales: mutuales, préstamos o créditos. No obstante, la vulnerabilidad social y económica de estos trabajadores es significativa, por tratarse de una actividad sin demasiadas perspectivas para los próximos años, aunque, de haber algún tipo de paralización, existen mecanismos de reinserción en otros establecimientos industriales por acuerdos intergremiales, la participación del gobierno y de los sectores empresariales. La fábrica de La Verde paralizó su producción en los últimos años y sus obreros fueron derivados a la taninera de La Escondida, ubicada a sólo $10 \mathrm{~km}$, hacia donde viajan diariamente con un transporte contratado por la empresa.

\section{La fábrica de mediana dimensión}

Tradicionalmente, los pueblos del Chaco tenían uno o más aserraderos (instalaciones que adquirían rollos o rollizos traídos desde los montes, que se encargaban de producir tablas, tablones, vigas y tirantes), que a su vez, eran los proveedores de estos insumos a un número variado de pequeñas carpinterías, las cuales se encargaban de fabricar -a pedido- los diferentes productos que demandaba la población local o del entorno (sobre todo aberturas y muebles). Esta modalidad aún perdura en 
gran parte de la provincia, excepto en el centro del Chaco, donde los establecimientos han integrado ambas funciones (aserraje y carpintería), en función de abaratar costos y de conformar una organización empresaria con capacidad de abastecer una demanda no sólo local, sino regional y, en muchos casos, de puntos más alejados del país. Este fenómeno, en forma todavía incipiente y puntual, se observa también en las grandes ciudades (Resistencia. Presidencia Roque Saenz Peña, Villa Angela y Charata).

En líneas generales, por el tamaño de las fábricas y la escala de producción que se observa en el Chaco, se prefirió utilizar el rótulo de "establecimientos medianos", ya que no existe la gran industria maderera que reúna caracteres de dimensión, tecnología y marketing como para situar los establecimientos en dicha categoría. Podemos clasificar estos establecimientos medianos en dos subgrupos: los mayoritarios, que demandan entre 60 y 120 tn de madera y disponen de alrededor de 7 empleados y 12 máquinas en promedio y, una minoría con capacidad de procesar más de 120 tn de madera, una media de 15 operarios y alrededor de 18 máquinas en funcionamiento.

La mayoría de estos fabricantes adquieren la madera de oferentes que las extraen de sus montes y, bajo algún arreglo con los transportistas, hacen llegar la materia prima (rollos o rollizos) a distintos lugares de la provincia, sobre todo al centro de la misma, donde se localiza la mayor parte de los aserraderos, carpinterías y mueblerías. Excepcionalmente, algunos industriales, a la vez dueños de campos con existencia de bosques nativos, utilizan los recursos maderables propios para su procesamiento; otras veces, gestionan algún tipo de acuerdo con otros propietarios de campos agropecuarios con disponibilidad forestal, para realizar la explotación de dichos recursos.

La producción de estos establecimientos se orienta fundamentalmente a la fabricación de uno o varios de los siguientes productos: muebles, artículos rurales, aberturas y tablones.

El industrial maderero típico dispone de experiencia, en muchos casos porque su padre y abuelo le enseñaron el oficio y, en otros, porque fue aprendiendo los gajes de la actividad como empleado hasta lograr la posibilidad de independizarse junto a su grupo familiar o algún socio. Quienes han podido alcanzar una escala de producción mediana, han contado con los recursos para participar de cierta capacitación, acceder a otros lugares para ampliar su campo de conocimientos o incorporar ciertas maquinarias, ya que en muy pocos casos podría hablarse de innovación tecnológica.

Estos productores no se hallan en situación de vulnerabilidad social, ni económica, son propietarios de sus viviendas, fábricas y equipamientos, disponen de medios de transportes, algunos de ellos tienen sus propios camiones para llevar la mercadería a los destinos de venta y gozan de un standard de vida que puede considerarse alto o medio alto a nivel de la sociedad chaqueña. Disfrutan de cobertura social, realizan aportes jubilatorios, poseen capacidad para adquirir bienes suntuarios y generar ahorros para costearse un período vacacional en verano junto a su grupo familiar en otros puntos del país e, incluso, en el exterior. También están en condiciones de adquirir o alquilar departamentos en otras ciudades para posibilitarles la formación universitaria a sus hijos, en los casos que ésas fueran sus expectativas.

\section{La fábrica de pequeña dimensión}

Alrededor del $60 \%$ de los establecimientos madereros están representados por instalaciones pequeñas que consumen mensualmente menos de 60 tn de rollos de madera, tienen una media de 5 operarios y alrededor de 7 máquinas.

En la mayoría de las pequeñas y medianas poblaciones del Chaco existen carpinterías y aserraderos que satisfacen las demandas locales. Por lo general, se trata de una actividad familiar (padres e hijos varones) y, en algunos casos, requieren uno o dos cadetes o empleados informales. El dueño, en el mejor de los casos, obtiene ganancias que le permiten poseer una vivienda propia, herramientas y máquinas para su emprendimiento y algún medio de movilidad: camión, tractor, camioneta o auto. En otros casos, el nivel de vida es inferior: el cuentapropista puede o no ser dueño de la casa que habita y del local donde desarrolla su actividad, sin disponer de vehículo. La mayoría no se halla registrado en organismos impositivos y son pocos los que aportan a una cobertura social y realizan aportes jubilatorios. Las pequeñas carpinterías, dedicadas a la fabricación de muebles y aberturas adquieren los tablones y tirantes de los aserraderos locales, suelen ser unipersonales o familiares y es común que desempeñan sus labores en el mismo predio donde tienen su vivienda. Su condición social y económica puede considerarse entre media y baja y no hacen aportes previsionales.

En las áreas de mayor concentración de establecimientos foresto-industriales, como Machagai, Quitilipi y Presidencia de La Plaza, ya se hizo referencia a muchas de las características propias -en 
las que subyace una cultura maderera- en el punto "Elevado dinamismo de los emprendimientos de pequeño tamaño". Debe considerarse que el grado de vulnerabilidad social y económica de estos emprendedores está entre medio y alto, dado que pueden sufrir la paralización por las causas ya enunciadas, pero, por otro lado, tienen la ventaja de reinsertarse rápidamente en el sistema, justamente por la existencia de un ámbito construido, donde el "saber hacer" representa la clave para acceder a un puesto de trabajo en otro establecimiento. Este fenómeno es bastante común en Machagai: un emprendedor puede pasar a ser empleado de otro establecimiento y, a la inversa, un asalariado, con el tiempo puede montar su pequeña empresa. Además, existe una gama de tareas que le permiten al desocupado temporario pasar el mal momento, como son las tareas de pulido, encerado y laqueado para las fábricas que demandan este servicio (trabajo que realiza en su propio domicilio o en lugares destinados a este fin) o la producción de artesanías.

\section{El sector asalariado (formalizados y no formalizados)}

El grupo de trabajadores que se halla en mejor condición es el que está registrado, es decir que, aparte de percibir el salario, cuenta con los aportes sociales, jubilatorios y cobertura de seguro por accidentes laborales, ya que se trata de una actividad con elevado riesgo, debido a la manipulación de máquinas cortantes como sierras, garlopas, cepilladoras, clavadoras y perforadoras. El segundo grupo, el más numeroso, se encuentra en una posición de alto riesgo en todo sentido: no dispone de cobertura alguna, constituye la variable de ajuste en épocas de menor demanda o de dificultades financieras de las empresas, por lo que puede quedar desocupado en cualquier momento; ante situaciones de accidentes, es frecuente que el patrón llegue a un acuerdo económico con el empleado, a fin de evitar acciones legales que se dilatan en el tiempo y que perjudican a ambas partes (al patrón por el elevado monto, en el que se suman intereses, tasas y vigorosos honorarios de abogados y, al empleado, por el largo tiempo de espera, a veces años, para el cobro de un dinero que también sufre un fuerte recorte por los servicios del letrado interviniente).

El perfil de un trabajador de aserraderos y carpinterías (formalizado o no), sobre todo del centro del Chaco, es el de un operario que ha aprendido el oficio de su familia o iniciándose como cadete en una fábrica, es decir que se ha formado observando y haciendo, con la orientación de sus patrones, capataces o compañeros de labor. De ninguna manera tiene un perfil técnico y sólo el paso de los años, sumado a su capacidad de superación lo hace más o menos eficiente dentro de la empresa. En términos generales, no tiene estudios secundarios completos $y$, en muchos casos, ni siquiera el primario y proviene de hogares de nivel socioeconómico bajo o medio-bajo. Son muy pocos quienes han accedido a cursos de capacitación o a entrenamientos específicos.

La ausencia de mano de obra calificada es un problema innegable dentro del sector maderero del Chaco. Un rasgo notable, sobre todo en el centro del Chaco, es el bajo nivel sociocultural de gran parte de los empleados de aserraderos y carpinterías -en su gran mayoría jóvenes-, que por su condición de informalidad incurre en ausentismos, especialmente después del fin de semana. En muchos casos, los mismos empleados se niegan a ser registrados, debido a que en los padrones figuran como desocupados y son beneficiarios de planes sociales que otorga el gobierno nacional, con lo cual al registrarse perderían dicho ingreso.

Si bien es difícil acceder a los datos cuando éstos tienen una alta tasa de no registrados, se estima que de la totalidad de empleados de aserraderos y carpinterías, menos del $40 \%$ de los mismos están en regla. A ellos deben sumarse los familiares del emprendedor que no están asentados en ningún registro, las pequeñas instalaciones que ni siquiera figuran como empresas a nivel municipal y que disponen de 1 a 5 operarios, incluido el dueño. Tampoco gozan de registro aquéllos que en los patios de sus casas, pequeños talleres y veredas de sus domicilios (a veces grupos familiares enteros o de parientes y amigos) realizan actividades de pulido y ensamblado de muebles o fabrican artesanías en forma permanente.

\section{El taller de pulido}

Esta es una actividad, a veces visible y, en la mayoría de los casos, invisible. La generalidad de los pulidores despliegan la actividad para fabricantes de muebles que les pagan por cantidad de trabajo realizado. El desplazamiento de los mobiliarios se acuerda con el fabricante: puede estar a cargo de éste (vehículo propio o contratado -en este último caso, por supuesto, de palabra) o el mismo pulidor, si tiene un carro u otro medio de movilidad, se encarga de este movimiento. Estas tareas no 
registradas se llevan a cabo en los domicilios del pulidor, a veces en un galpón o bajo un simple techo, o sencillamente a la sombra de algún árbol. Se trata de una actividad muy azarosa, ya que es mucha la gente que se ofrece para estas tareas (elevada competencia) y depende de la intensidad de la demanda que tienen las carpinterías. Por ejemplo, entre diciembre y marzo (época de vacaciones) se observa una fuerte retracción en la demanda de muebles y, lo mismo ocurre durante períodos lluviosos en los que escasea la materia prima y, por tanto, se reduce la producción. La vulnerabilidad social y económica de estos trabajadores independientes y de sus núcleos familiares es alta, al no tener ingresos más allá de los necesarios para el sustento diario, carecer de cobertura social y otros aportes y, además, estar expuestos a riesgos de salud por accidente o aspiración de polvillo u otras partículas sólidas cuando trabajan con lacas que luego son lijadas o pulidas.

\section{La intermediación: acopiadores y revendedores}

Básicamente, esta modalidad se da en la industria del mueble. Quienes se dedican a esta actividad intermediaria suelen tener distintos orígenes: personas de las comunidades locales que están o estuvieron vinculadas con la industria maderera, ya sea como dueños, socios o empleados de aserraderos o carpinterías; aquéllos que, sin haber estado relacionados con la actividad, han reunido el capital inicial necesario y los contactos suficientes para colocar los productos en otros puntos del país y, finalmente, quienes arriban de otras provincias para desarrollar puntualmente esta función. Muchos de los acopiadores y revendedores no residen en la provincia, pero mensualmente viajan a la misma para concretar los negocios o tienen un representante local en el área de producción; cuando logran reunir un volumen que les permita completar un equipo de transporte, se procede al traslado de los productos. Estos acopiadores externos se localizan en Santiago del Estero, Córdoba, Santa $\mathrm{Fe}$, Buenos Aires y en otras provincias argentinas. Los actores que intervienen en este negocio, en general, no tienen dificultades de orden económico y, por tanto, están fuera de los grupos vulnerables desde el punto de vista social y económico. Los camiones utilizados para conectar la oferta con la demanda pueden ser propios o de terceros que son contratados para este servicio; en casos puntuales, pueden pertenecer al propio establecimiento foresto-industrial, el cual se encarga de colocar su producción en los lugares de destino.

Una variante en el acopio y reventa, pero en condiciones sociales y económicas muy diferentes, se da con los productos artesanales: muchas personas constituyen puestos de ventas informales, sobre todo a la vera de la ruta nacional $N^{\circ} 16$, sin ser artífices de la madera y lo hacen adquiriendo las manufacturas o por medio de un acuerdo económico con el artesano, que logra así darle salida a su producción. Este actor sí se encuentra en condiciones socioeconómicas desfavorables, ya que su trabajo tiene mucho de imprevisibilidad y su grado de vulnerabilidad puede ser alto ante diferentes circunstancias (crisis económicas, cortes de rutas o piquetes, controles municipales o impositivos, aumento de la competencia). Como no es una actividad registrada, suelen ser beneficiarios de planes sociales.

\section{La producción artesanal}

La fabricación de artesanías fue inicialmente una actividad puntual y poco relevante, pero con el paso del tiempo y, particularmente desde el año 2008 , se ha observado un gran crecimiento, tanto en el número de artesanos, como en los tipos y calidad de diseños de sus productos, a tal punto que la mayoría de ellos se nuclean en una cooperativa que les permite organizar exposiciones en distintos lugares de la provincia y el país. El crecimiento explosivo de los últimos años se dio a partir de algunos artesanos y revendedores que se instalaron a orillas de la ruta nacional $N^{\circ} 16$ al aire libre 0 debajo de algún árbol situado en cercanías de las banquinas, permitiéndole al viajero conocer y adquirir estos productos regionales. El éxito de estos visionarios fue el detonante para que otros los imitaran rápidamente, formándose así largas líneas con puestos de ventas a ambas márgenes de la mencionada vía de circulación, sobre todo en Machagai.

El artesano típico desarrolla su actividad en forma individual o con la participación de su grupo familiar, sobre todo en la comercialización de sus productos. Muchos de ellos trabajan para un comprador fijo, generalmente foráneo, donde coloca la totalidad de la producción, asegurándose una renta mensual mas o menos fija, aunque en este caso, como toda venta a granel, debe bajar el costo por unidad, pero como contrapartida, recepciona en una sola operación comercial un monto en efectivo que le da cierto alivio a su economía familiar. 
El reciente impulso de esta actividad es altamente positivo para reducir la entropía del poco eficiente sistema maderero, dado que utiliza un recurso que tradicionalmente fue de desperdicio o de muy baja remuneración (costaneros, astillones, rodajas) como leña de los hornos de las ladrillerías o carbonerías de la zona.

El artesanado pertenece a la economía netamente informal; no se hallan registrados en ningún organismo municipal, provincial o nacional y, por lo tanto, no disponen de obra social, ni hacen aportes jubilatorios, estando fuera del esquema laboral oficial. No obstante, amparados en esa informalidad, la mayoría de ellos o su grupo familiar reciben beneficios sociales como planes sociales, becas u otras modalidades.

\section{El obtención de leña}

La extracción de leña del bosque chaqueño admite al menos cuatro modalidades, según el destino que se le dé a este recurso: a) para autoconsumo de la población rural pobre (esta ha sido una práctica tradicional desde el inicio de la colonización, con una tendencia restrictiva debido a la emigración demográfica sufrida por el campo en los últimos 50 años y la difusión del gas en garrafa como combustible; b) como materia prima de los hornos de carbón; c) para venta directa (destinada a calderas de máquinas de ferrocarril hasta la década del '60 y para el consumo de hornos industriales como panaderías y pequeñas fábricas de diferentes insumos o productos que no requieren altos niveles de energía calórica; d) para consumo de ladrillerías.

Una quinta opción sería la posibilidad de abastecer a una fábrica de arrabio brasileña con 100.000 tn anuales de leña. El gobierno de la provincia del Chaco llevó a delante esta iniciativa, junto a empresarios del Brasil (firma Vetorial) para su instalación en Puerto Vilelas (Gran Resistencia) en el predio de la ex fábrica de arrabio TAMET S.A. (Talleres Metalúrgicos Sociedad Anónima) que funcionó en los años '70. Las obras se hallan en ejecución, para comenzar a producir en el primer semestre del 2012. El proyecto prevé la obtención de arrabio durante los primeros tres años, hasta llegar a niveles de producción que permitan la fabricación de acero y, con los desechos, la fabricación de cemento. Se ha estimado que esta planta ocupará a 400 empleados directos y 200 indirectos al llegar a la segunda fase (producción siderúrgica). Esta instalación industrial generará valor agregado y empleo formal. Esta decisión causó bastante malestar en la comunidad vilelense y en otros estamentos de la sociedad chaqueña, por los efectos ambientales, tanto de la deforestación como de la actividad industrial, pero de todos modos, el proyecto ha sido impulsado desde la política provincial y nacional y parecería no detenerse.

\section{La fabricación de postes}

Existen aserraderos dedicados a la elaboración de artículos rurales que producen postes y varillas, entre otros productos. Pero también hay hacheros que, dentro de una diversidad de actividades periféricas en el medio rural, se dedican a la fabricación de postes de quebracho colorado que ellos mismos ofrecen a la venta de manera informal o por pedido específico de ganaderos o agricultores de la zona. La extracción de la madera se hace en tierras fiscales, en áreas sin dueños identificados o en acuerdo con el propietario de montes, donde se realiza la entresaca y ambos tienen beneficios económicos. Este trabajador informal presenta condiciones desfavorables y alto grado de vulnerabilidad social y económica.

\section{La producción de carbón de leña}

El Chaco, históricamente fue una de las principales provincias exportadoras de carbón vegetal. Se reconocen dos grupos, por un lado, los productores de mediana escala, que generalmente son propietarios de aserraderos, quienes derivan los desperdicios de su industria a esta actividad para darle mayor eficiencia económica a su empresa; también en este grupo se encuentran aquéllos que hacen del carbón su actividad principal, disponiendo de un número considerable de hornos (más de cinco) al cuidado de personal no registrado, cuya producción venden con una frecuencia estipulada (quincenal o mensual) a equipos que provienen de la propia provincia o de otros lugares del país. Una parte de estos productores están registrados, por ser empresarios madereros. Por otro lado, se halla el productor que dispone de uno o más hornos en tierras que generalmente ocupa (del fisco, de propietarios no bien identificados o de calles muertas) o donde ha logrado hacer algún arreglo 
informal con el propietario de tierras y montes que reditúe en beneficio económico para ambas partes. Esta es una labor de subsistencia y los escasos volúmenes de producción se venden a acopiadores o a los comercios de la zona para su reventa. Esta actividad es totalmente informal, es decir, no se halla registrada en ningún organismo del Estado y, por ende, los grados de vulnerabilidad social y económica de estos trabajadores son elevados.

\section{La empresa de servicios diversificados}

Corresponde a los comercios que proveen herramientas, máquinas e insumos a los aserraderos y carpinterías. Por lo general son ferreterías tradicionales que han ido adaptando su oferta a las necesidades exigidas por el sector foresto-industrial, sobre todo en el centro y en las ciudades más jerarquizadas de la provincia. En los últimos años se han sumado nuevas casas de ventas y, algunas de ellas, ofrecen el servicio de mantenimiento de equipos y afilado de sierras. Estas actividades conexas agrupan a personas y familias de condición económica y social entre media y alta, sin riesgos de vulnerabilidad, que al mismo tiempo generan trabajo, ya que emplean a operarios, choferes y vendedores para ofrecer estos servicios.

\section{La fabricación de ladrillos}

El típico "ladrillero" desarrolla esta actividad en tierras marginales suburbanas o rurales que ocupa o alquila, en un contexto de pobreza general. Realiza este trabajo junto a su grupo familiar o personas asociadas de hecho (parientes, amigos o vecinos), representando una tarea de subsistencia marginal e informal, cuya comercialización raramente supera la escala local. Para la venta puesta en obra suelen disponer de carritos tirados por caballos o recurren del servicio de terceros. No siempre se dedica a tiempo completo, sino que la complementa con changas o con actividades agropecuarias de subsistencia (cultivos, cría de cabras o cerdos o producción de leña). Suelen ser beneficiarios de planes sociales, familia numerosa y es muy común que sus mujeres, por predominar el vínculo de concubinato, cobren aportes por madres solteras.

Las ladrillerías conforman instalaciones bastante precarias y funcionan en buena medida como actividades conexas de la industria maderera (uso de cascarilla, costaneros y aserrín). Las extracciones de suelos aptos para uso urbano o rural, así como la generación de contaminantes (humo) influyen directamente reduciendo la calidad de vida de los propios productores y de los vecinos, quienes pueden concentrarse en barrios muy poblados. Los sitios próximos a las ladrillerías comúnmente albergan a los grupos familiares dedicados a estas tareas y se caracterizan por la precariedad de las viviendas, por el deficiente estado sanitario debido a la falta de infraestructura y servicios básicos y, lógicamente, los índices de NBI (Necesidades Básicas Insatisfechas) son elevados. Este grupo es el que, en términos generales, se halla expuesto a los mayores riesgos, no sólo económicos, sino sanitarios y ambientales. Es, indudablemente, el sector que sufre el mayor grado de vulnerabilidad social y económica dentro de la cadena vinculada al aprovechamiento de los recursos madereros.

\section{El sector transportista}

Durante más de medio siglo (entre 1909 y principios de la década del '70) existió una fuerte demanda de maderas chaqueñas para uso ferroviario. Por un lado, de leña para alimentar las calderas de los motores de los trenes y, por otro, de durmientes (principalmente de quebracho colorado) para usarlos como bases de apoyo de los rieles de acero que constituyen las vías, tanto las que se emplazaron en el Chaco, como en otros puntos del país. La gran demanda se produjo durante la etapa de expansión del ferrocarril, aunque posteriormente se continuaron requiriendo maderas duras para el mantenimiento de las vías. Con la aparición de máquinas diesel esta demanda se redujo hasta desaparecer.

Una segunda participación del sector transportista está vinculada con el traslado de rollos, rollizos, leña, maderas aserradas, carbón vegetal y productos manufacturados e industrializados. El camión ha garantizado la conectividad entre las áreas forestales, los puntos o áreas de procesamiento y los lugares de destino de la producción. Aquí participan varios actores, entre los que están: a) los empresarios del transporte: personas o sociedades que disponen de flotas de camiones y equipos que son contratados por diferentes rubros de la actividad económica, entre ellos el sector maderero; 
b) los propietarios de uno o más camiones que se dedican al transporte en general o específicamente al sector maderero; c) los dueños de aserraderos/carpinterías que disponen de camiones y que incorporaron este servicio; d) los empleados que se desempeñan como choferes, que son asalariados que dependen de cualquiera de los grupos empleadores citados en los ítems anteriores. En líneas generales, ninguno de ellos se enmarca en situaciones de vulnerabilidad social o económica; y, finalmente, e) los carreros, que ocupan el extremo de esta cadena, complementándola con una diversidad de changas, en las que participa el grupo familiar, incluidos los niños. Como es esperable, los grados de vulnerabilidad social y económica son muy elevados en este grupo.

\section{La explotación de recursos forestales}

En el Chaco, la obtención de maderas para diferentes usos siguió tres caminos durante gran parte de la historia: la autorización o concesión del Estado a un particular o sociedad para llevar a cabo el aprovechamiento del recurso en tierras fiscales; la explotación por parte del propio dueño de las tierras forestales y la autorización de éste para que un tercero lleve a cabo la entresaca o tala rasa en su propiedad. De hecho, durante mucho tiempo se ha dado el fenómeno de explotación de los recursos forestales de tierras fiscales sin que mediara autorización, situación que en los últimos años se ha ido restringiendo, debido a los cambios en las normativas legales y a un mayor control estatal que penaliza fuertemente al infractor, tanto a quien desmonta como a quien transporta maderas ilegalmente. Con la nueva legislación, todo uso maderable del bosque chaqueño debe ser autorizado por las autoridades pertinentes, previo estudio de impacto ambiental. Lamentablemente, en la década del '90 se han perdido enormes extensiones de bosques en el sudoeste (desmonte y quema) para abrir paso a la producción sojera, ante la ausencia o complicidad del Estado; en el noroeste, también hubo una explotación intensiva de ciertas áreas, cuyas maderas en parte abastecían la demanda de aserraderos y carpinterías de la provincia y, por otra, eran sacadas hacia Santiago del Estero (rollos, rollizos y postes) con destino a diversas provincias argentinas. Las condiciones socioeconómicas de los actores encargados de la explotación forestal ha sido muy variable en el espacio y en el tiempo, ya que incluye al pequeño productor que logra un mínimo ingreso (único o discontinuo) para inyectarlo a su alicaída economía, hasta el gran terrateniente que percibe sumas millonarias por uso propio o concesión de sus recursos maderables. El grado de vulnerabilidad, como puede evidenciarse, puede ser significativo en un extremo y nulo en el otro. Lo que es innegable es que los negocios ilegales de la madera han dejado abundantes dividendos a numerosos empresarios inescrupulosos, tanto locales como foráneos.

\section{Los sectores potenciales}

Se le da este nombre a aquéllos emprendimientos que aún no están en marcha, pero que tienen cierta probabilidad de desarrollarse en la provincia. El más avanzado, en estado de ejecución de obras es el de la citada instalación de una fábrica de arrabio en Puerto Vilelas que, se estima, le impregnará un importante dinamismo al trabajo de obrajes, dueños de montes y transportistas.

Otras actividades industriales que se encuentran aún en experimentación en escala reducida o en estado de proyecto son la fabricación de pellets (para generar energía calórica), espirales y madera aglomerada a partir de los residuos o madera de descarte de los aserraderos y carpinterías.

El siguiente cuadro ilustra la importancia del sector maderero en las tres localidades del centro del Chaco que concentran el grueso de la actividad, sin considerar transportistas (dueños de camiones, choferes de camiones, tractores y grúas), ni motosierristas:

Personal registrado y no registrado por subtipos de actividades en la industria maderera centrochaqueña.

\begin{tabular}{|l|l|l|l|l|}
\hline & Machagai & Quitilipi & P. de La Plaza & Total \\
\hline Dueños, socios, gerentes & 375 & 245 & 75 & 695 \\
\hline Aserraderos y carpinterías & 1.700 & 884 & 340 & 2.924 \\
\hline $\begin{array}{l}\text { Talleres pulido, ensamblado, } \\
\text { afilado }\end{array}$ & 100 & 20 & 5 & 125 \\
\hline Artesanos & 30 & 10 & 2 & 42 \\
\hline Revendedores & 15 & 10 & 5 & 30 \\
\hline Carboneros & 40 & 50 & 10 & 100 \\
\hline
\end{tabular}

* Valores aproximados sobre la base de relevamientos in situ. Elaboración propia. 
Como se observa en el cuadro, unas 4.000 personas estarían directamente involucradas con la industria maderera en estas tres localidades, equivalente al 5,7\% de la población total, aunque si agregamos al núcleo familiar de estos actores, en conjunto representarían no menos del $25 \%$ de los habitantes de estas comunidades. Discriminando por localidad, a los efectos de saber el peso relativo de la actividad en cada centro urbano, puede decirse que en Machagai el 8,4\% de la población se desenvuelve en este rubro y a nivel del grupo familiar en dependencia de la actividad el valor ascendería a un 33\%; Quitilipi estaría en 4,2\% y $17 \%$ respectivamente y Presidencia de la Plaza en $3,5 \%$ y $14 \%$, siguiendo el mismo orden de distribución. Como se desprende de este análisis, Machagai (conocida como el polo maderero del Chaco) es la ciudad con mayor dependencia de la actividad, al tener involucrada en ella a $1 / 3$ de su población, en tanto Quitilipi y Presidencia de la Plaza participan con valores absolutos y relativos bastante menores.

\section{Conclusiones}

Los recursos forestales han tenido un rol decisivo en la historia del Chaco, tanto en los aspectos demográficos como en las trazas de las vías de transporte y comunicación y en la dinámica misma de su economía. La incidencia de la actividad taninera es harto conocida durante las primeras décadas del siglo XX y todavía se observan sus relictos en la geografía chaqueña. Aún hoy, pasados más de 130 años de los primeros obrajes instalados cerca del río Negro en la franja oriental, la actividad maderera sigue teniendo vigencia y una influencia directa sobre los distintos actores que se movilizan sobre un escenario siempre dinámico, donde existen sectores que ganan y otros que pierden. Desde una visión crítica, estas dicotomías han impregnado al sector foresto-industrial desde sus comienzos: blancos y criollos desplazando a los habitantes originarios para hacer uso de los recursos forestales que constituían su hábitat; empresarios transnacionales ricos versus empleados miserables en la etapa de auge de las fábricas de tanino; fabricantes, transportistas, empresarios forestales e intermediarios prósperos frente a pequeños productores, operarios informales, carboneros y ladrilleros pauperizados que luchan entre la subsistencia y las necesidades básicas insatisfechas, la pobreza y la vulnerabilidad social y económica, a la par de la degradación de los ambientes que constituyen las bases de su sustento.

Sin dudas, la explotación forestal y la actividad industrial vinculada a la misma se caracterizan por la visibilidad de ciertos componentes (plantas procesadoras, aserraderos, carpinterías, salones de exposición y ventas, artesanos, camiones que transportan maderas y productos elaborados) y la invisibilidad de muchos otros (pulidores, fabricantes de postes, de carbón vegetal, leña o ladrillos) que no están registrados en ningún lado, cuyo trabajo vale muy poco y casi siempre llevan las de perder (de hecho, para el sistema impositivo del Estado, estos trabajadores se mueven en el marco de la ilegalidad).

Conocer la constitución interna de la trama forestal-industrial nos lleva a identificar las desigualdades existentes entre los distintos actores y detectar que algunos de ellos están expuestos a grados de vulnerabilidad muy elevados, al tiempo que su conectividad a la red formal y visible pende de un hilo muy débil, susceptible de cortarse ante cualquier situación anómala o imprevista. Las autoridades gubernativas deberían dejar de ver a estos ciudadanos como actores ilegales de un escenario dominado por personajes visibles y, en contrapartida, generar las condiciones necesarias para posibilitarles su participación bajo el amparo de dispositivos legales y mecanismos de inclusión (políticas crediticias para impulso de microemprendimientos, asistencia social a los grupos familiares, exenciones impositivas a los pequeños productores, cursos gratuitos de oficios para jóvenes emprendedores, fomento de pequeñas cooperativas y asesorías comerciales para la colocación de sus productos en el mercado). 


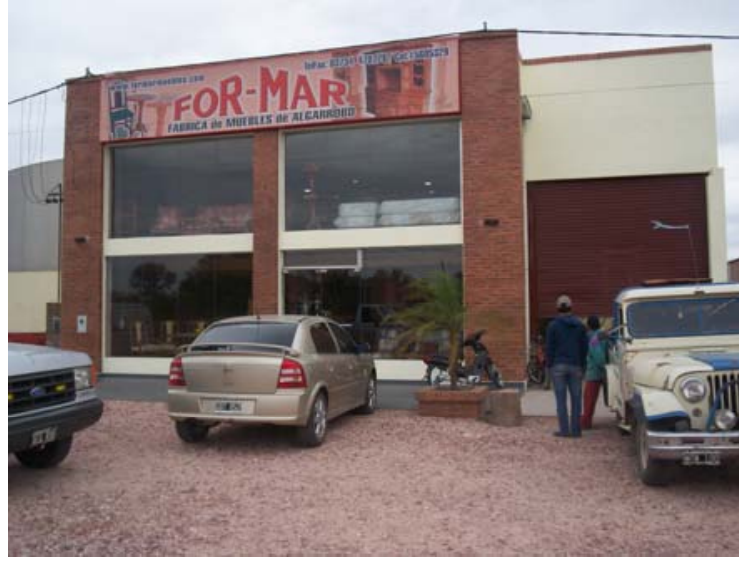

Aserradero, carpintería y salón de venta de muebles en Machagai, sobre ruta nacional 16.

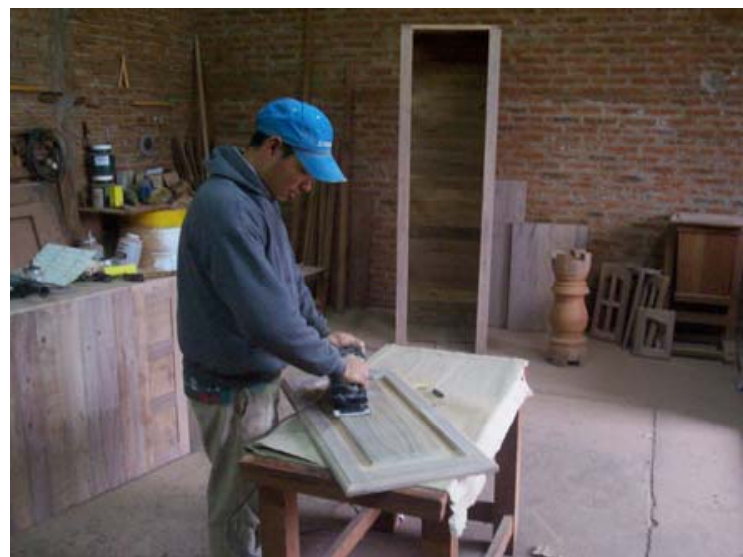

Pulidor de muebles: una actividad muy extendida en el centro del Chaco

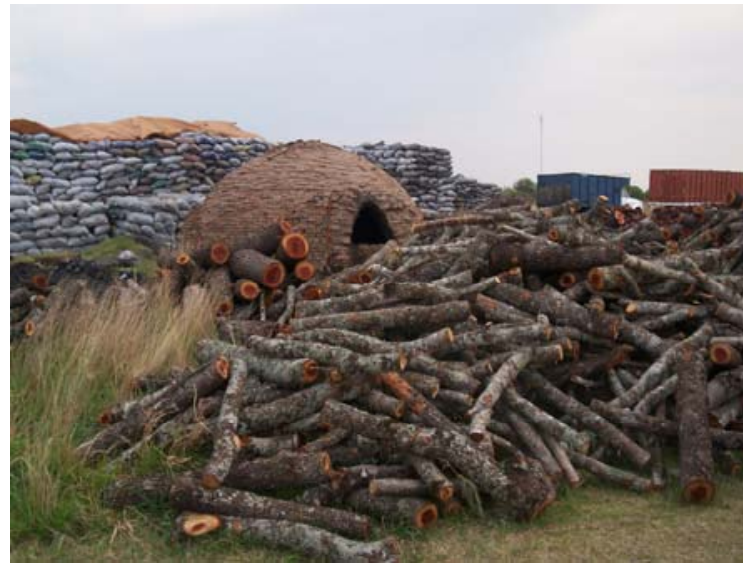

La producción de carbón aprovecha la leña y los sobrantes de madera de los aserraderos.

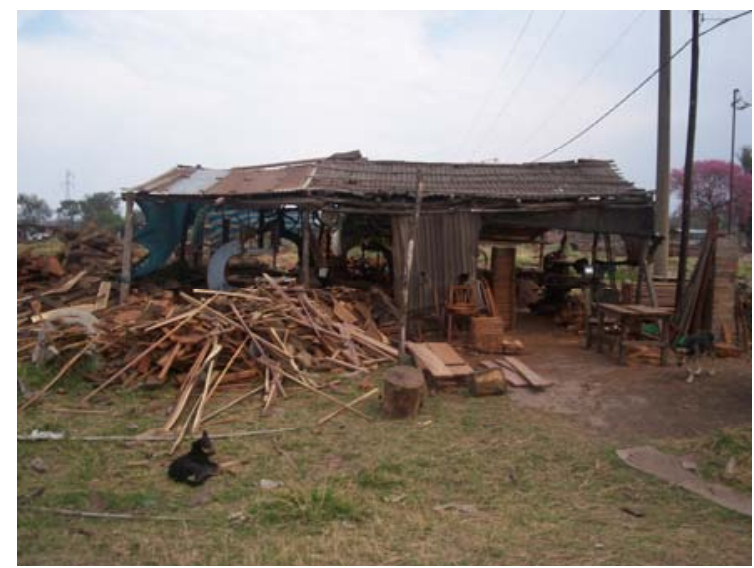

Pequeño aserradero en Presidencia de La Plaza.

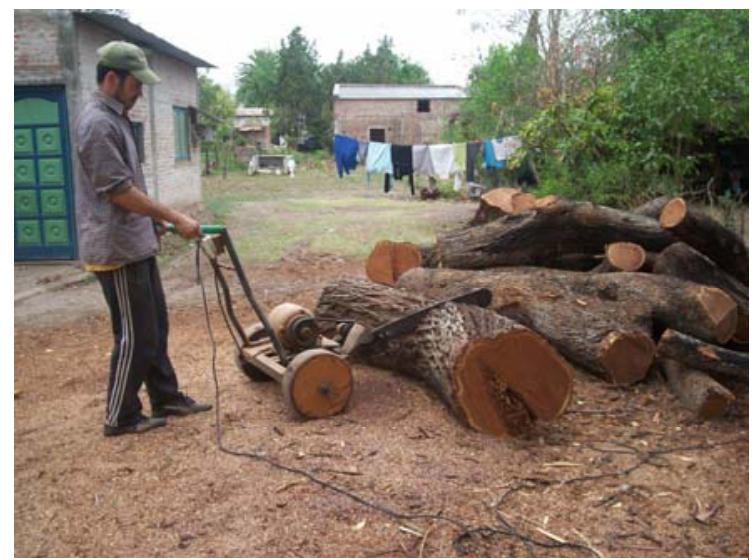

El pequeño fabricante de muebles y artesanías desarrolla su actividad con escasa tecnología.

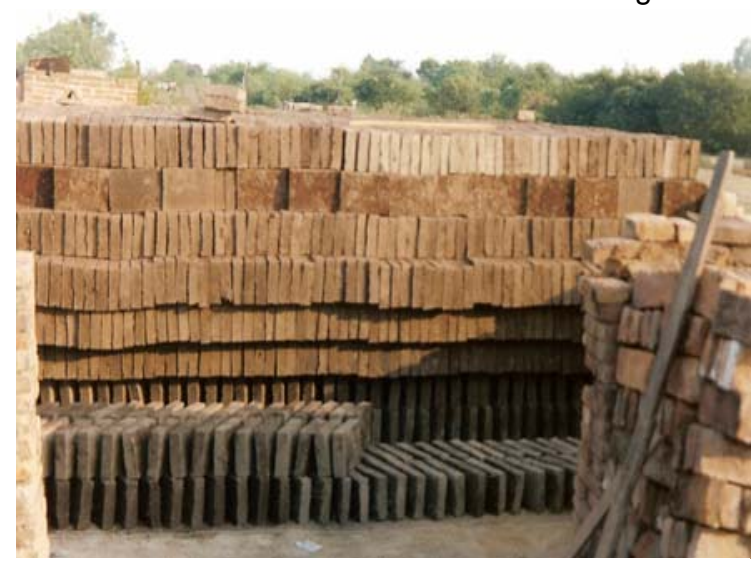

La producción de ladrillos: demanda leña y costaneros, que provienen de los aserraderos, para las horneadas 
Revista Geográfica Digital. IGUNNE. Facultad de Humanidades. UNNE. Año 8. № 15. Enero - Junio 2011.

\section{Materiales consultados}

Altamirano, M. 1999. Historia de la industria taninera en La Verde (Chaco). Folia Histórica del Nordeste No 14. IIGHI - CONICET, Instituto de Historia (UNNE). Resistencia. Pp. 63 a 94.

Astori, R. E. 1997. El manejo del recurso forestal en la provincia del Chaco. Fac. Arquitectura y Urbanismo. UNNE. Inédito. Resistencia.

Basail, M. A. 1995. Análisis y diagnóstico del estado actual del quebracho colorado chaqueño. Fac. Arquitectura y Urbanismo. Inédito. UNNE. Resistencia.

Beck, H. 1997. Pueblos del Chaco: el poblamiento del territorio a partir de la formación de núcleos urbanos 1878-1950. Revista Nordeste. Segunda Época. Docencia: Historia, № 3. Facultad de Humanidades - UNNE. Resistencia.

Besil, A. 2001. La economía del Chaco en la década de los '90. Rev. Indicadores Económicos No 44. Resistencia, 2001.

Beveraggi, M. 1995. Análisis del bosque chaqueño como recurso natural. Fac. Ciencias Exactas y Naturales y Agrimensura. Fac. Arquitectura y Urbanismo. UNNE. Inédito. Corrientes - Resistencia.

Bialet Massé, J. 1904. Informe sobre el estado de las clases obreras en el interior de la República. Buenos Aires. Tomo I. Pp. 30-45.

Borrini, H. R. 1999. Los efectos de la industria en el poblamiento del Territorio Nacional del Chaco 1878-1950. Folia Histórica del Nordeste No 14. Resistencia. Pp. 95 a 120.

Braier, G. 2004. Informe Nacional Complementario. Argentina: tendencias y perspectivas del sector forestal del año 2020. Secretaría de Desarrollo Sustentable y Política Ambiental. Buenos Aires.

Bruniard, E. D. 1978. El Gran Chaco Argentino. Revista Geográfica No 4. Instituto de Geografía. Universidad Nacional del Nordeste. Resistencia.

Bunge, A. 1922. Las industrias del norte. Contribución al estudio de una nueva política económica argentina. Volumen I. Buenos Aires.

Bünstorf, J. 1982. El papel de la industria taninera y de la economía agropecuaria en la ocupación del espacio chaqueño. Folia Histórica del Nordeste $N^{0} 5$. Instituto de Historia -Facultad de Humanidades - UNNE e Instituto de Investigaciones Geohistóricas - CONICET. Resistencia, Corrientes. Pp. 7 a 59.

Cassagne Serres, A. 1929. La industria forestal argentina. Obrajes, explotación, comercio, contratos de arrendamientos, tasaciones de bosques, operaciones, contabilidad. Buenos Aires. CeDeTeMa (Centro de Desarrollo Tecnológico de la Industria de la Madera): Informes de difusión. Machagai, Chaco, 2005.

Cozzo, D. 1962. La industria forestal argentina. Cuadernos de Cicso. Serie de Estudios № 11.Buenos Aires.

Cuadra, D. 2008-2010. Base de datos. Elaboración propia a partir de trabajos de campo y entrevistas a actores calificados. Años 2008-2010.

Cuadra, D. 2008. La industria maderera en el área centrochaqueña. Situación actual y perspectivas. Conferencia. XII Encuentro de Profesores en Geografía. Universidad Nacional del Nordeste. Resistencia, Argentina. Fecha: 19-09-2008.

Cuadra, D. 2007. Makallé. Lucha y existencia de un pueblo chaqueño. Compendio Geohistórico. Ediciones Moglia S.R.L. Corrientes.

Dandan, A. 2008. Explotación forestal. Diario Página 12. Fecha: 06 de junio de 2008. Buenos Aires. Daus, F. 1940. Los bosques del Chaco. Revista Humanidades XXVIII. La Plata.

Del Valle Curi, M. 1968. Industria forestal en el Chaco. Asociación de Productores Forestales del Chaco. Resistencia.

Diario Norte. 2009. El veto a la Ley 6409 no debilita la protección de los bosques nativos. Resistencia Chaco. 01 de noviembre de 2009. Pp. 7.

Diario Norte 2008. El sector maderero firmó convenios con la provincia. Fecha: 10 de setiembre de 2008. Resistencia, Chaco. Pp. 34.

Diario Norte 1999. Producción material de Machagai. Entrevistas a actores de la actividad y comentarios sobre la Feria Provincial de la madera, parque industrial y comercialización. Fecha: 17 de diciembre de 1999. Resistencia, Chaco. Pp. 22 y 23.

Diario Norte 2008. Suplemento Foresto Industrial. Fecha: 25 de setiembre de 2008. Resistencia, Chaco. Pp. 15, 16 y 33. 
Diez de los Ríos, S. 1998. Proceso de deforestación del bosque chaqueño. Fac. Arquitectura y Urbanismo. UNNE. Inédito. Resistencia.

El Diario 1997. Culmina la fiesta provincial de la madera en Machagai. Fecha: 14 de diciembre de 1997. Resistencia, Chaco. Pp. 6 y 7.

Cuadra, D. 2010. Entrevistas a informantes calificados vinculados directa e indirectamente con la actividad foresto-industrial. Inédito. Años 2008-2010.

Cuadra, D. 2008. Entrevista al productor maderero Mario Ramirez (Machagai, Chaco), Inédito. 11 de agosto de 2008.

Feleniuk, A. N. 1998. Síntesis histórica de la explotación forestal en la provincia del Chaco: algunas propuestas. Fac. Arquitectura y Urbanismo. UNNE. Inédito. Resistencia.

Franceschini, M. C. 2001. El bosque de General Güemes: un caso más de explotación irracional del bosque chaqueño. Algunas propuestas para el uso sustentable. Fac. Arquitectura y Urbanismo. UNNE. Inédito. Resistencia.

García Pulido, J. 1975. La explotación del quebracho e historia de una empresa: la tragedia de nuestros hacheros. Casa García. Resistencia.

Girbal de Blacha, N. M. 1993. Explotación forestal, riesgo empresario y diversificación económica: las inversiones argentinas en el Gran Chaco (1905-1930). Revista de Historia de América No 116. Instituto Panamericano de Geografía e Historia. México.

Gobierno de la Provincia del Chaco. 2006. Inventario Forestal 2005: Provincia del Chaco. CDROM. Resistencia.

Gori, G. 1974. La Forestal: la tragedia del quebracho colorado. Proyección. Resistencia.

Maeder, E. 1996. Historia del Chaco. Ed. Plus Ultra. Buenos Aires.

Miranda, G. 1980. Tres Ciclos Chaqueños (Crónica Histórica Regional). Nordeste Impresora. Resistencia.

Núñez de Correa, R. 1996. Fábrica taninera Puerto Tirol: su impacto ambiental. Fac. Arquitectura y Urbanismo. UNNE. Inédito. Resistencia.

Ospital, M. S. 1990. Condiciones laborales en la explotación forestal del Gran Chaco Argentino. Folia Histórica del Nordeste No 9. IIGHI, CONICET - UNNE. Resistencia.

Piñeiro, D. y Caracciolo, M. 1974. Historia Económica y Social del Chaco. Cuadernos de Cicso. Serie Estudios $N^{\circ} 11$. Buenos Aires.

Ramírez, M. 1983. La expansión ferroviaria de origen forestal. Boletín del Instituto de Geohistoria. IIGHI - CONICET. Resistencia.

Ruberto, A. R. 1996. La Forestal como empresa multinacional. Fac. Arquitectura y Urbanismo. UNNE. Inédito. Resistencia.

Schaller, E. 1986. La colonización en el Territorio Nacional del Chaco en el período 1869-1921. C.G.R. No 12. IIGHI. Resistencia.

Schmid, L. F. 2001. Desmonte y recuperación de los bosques nativos de la provincia del Chaco. Fac. Arquitectura y Urbanismo. UNNE. Resistencia.

Sirvén, R. R. 2005. Bosque, industria y capacitación en el Chaco. Secretaría de Agricultura, Ganadería, Pesca y Alimentación. № 34. Buenos Aires. Pp. 17 a 20.

Zarrilli, A. 2000. Transformación ecológica y precariedad económica en una economía marginal. El Gran Chaco Argentino, 1890-1950). Revista Theomai. Estudios sobre Sociedad, Naturaleza y Desarrollo. № 1.

Zorrilla, A. 2005. Proyecto Competitividad y Medio Ambiente. Informe de avance. Machagai, 2005. 